\title{
The association between hippocampal subfield volumes and education in cognitively normal older adults and amnestic mild cognitive impairment patients
}

This article was published in the following Dove Press journal:

Neuropsychiatric Disease and Treatment

\section{Dong Woo Kang' \\ Hyun Kook Lim² \\ Soo-hyun Joo' \\ Na Rae Lee' \\ Chang Uk Lee'}

'Department of Psychiatry, Seoul St Mary's Hospital, College of Medicine, The Catholic University of Korea, Seoul, ${ }^{2}$ Department of Psychiatry, Yeoui-do St Mary's Hospital, College of Medicine, The Catholic University of Korea, Seoul, Republic of Korea
Correspondence: Chang Uk Lee Department of Psychiatry, Seoul St Mary's Hospital, The Catholic University of Korea, 222 Banpo-daero, Seocho-gu, Seoul I37-70I, Republic of Korea

Tel +82 222586082

Fax +82 25368744

Email jihan@catholic.ac.kr
Objective: Previous research has indicated that there are potential associations between education and total hippocampal volume in the trajectory of Alzheimer's disease (AD). However, the correlation between education and hippocampal subfield volumes in the progression of AD has yet to be understood. This study examined the relationship between education, which is a standard proxy for cognitive reserve, and hippocampal subfield volumes in healthy and amnestic mild cognitive impairment (aMCI) groups.

Subjects and methods: Thirty-eight subjects with aMCI and 39 healthy control subjects underwent 3 T magnetic resonance imaging, and hippocampal subfield volumes were measured by automated segmentation. Multiple linear regression analysis was used to determine the association between education and hippocampal subfield volumes.

Results: Education had a significant negative correlation with the left parasubiculum, presubiculum, and subiculum volumes in the aMCI group. In addition, multiple subfield volumes including left parasubiculum, left/right presubiculum, left cornus ammonis (CA)3, and left CA4 showed a significant correlation with the neuropsychological test scores in the control group and aMCI group.

Conclusion: These findings contribute to a better understanding of the association between education, hippocampal subfield volumes, and amnestic cognitive functions in the early phase of $\mathrm{AD}$.

Keywords: education, hippocampus, presubiculum, subiculum, mild cognitive impairment, cognitive reserve

\section{Introduction}

Education is one of the known modifiable factors which modulate the course of Alzheimer's disease. ${ }^{1}$ Low level of education has been documented to be related with increased prevalence of Alzheimer's dementia (AD). ${ }^{2}$ In addition, meta-analyses of education have shown higher education has a protective role against developing $\mathrm{AD}$ and delays the progression of AD due to tolerance of increased AD pathology. ${ }^{3}$ Furthermore, previous studies have demonstrated that education is correlated with the structural and functional changes of hippocampus, which is vulnerable to the pathogenesis of $\mathrm{AD},{ }^{4,5}$ in healthy older adults, mild cognitive impairment (MCI) and AD patients. ${ }^{6-8}$

Among these changes of hippocampus, hippocampal volume loss has been associated with the early progression of AD. ${ }^{9}$ However, some controversy exists concerning the relationship between education and hippocampal volume in the progress of AD. 
Although several studies comparing amnestic MCI (aMCI) and $\mathrm{AD}$ patients with healthy controls have shown a nonsignificant relationship between education and hippocampal atrophy, ${ }^{6,10}$ another longitudinal study of cognitively normal older adults found a significant relationship between mental activity including education and the rate of hippocampal atrophy. ${ }^{11}$

Because the hippocampus is a heterogeneous structure consisting of interacting subregions, ${ }^{12}$ recent studies have reported selective changes in volumes within the subregions of the hippocampus measured in vivo with magnetic resonance imaging (MRI) during the trajectory of $\mathrm{AD} \cdot{ }^{13-15}$ These hippocampal subfields can be delineated by manual or automated segmentation. Van Leemput et al introduced an automated segmentation technique based on a parametric method modeling the spatial distribution of the hippocampal subfields to overcome the limitations of manual segmentation, which is labor intensive and time consuming. ${ }^{16}$

However, there are few reports relating education to hippocampal subfield volumes in the progression of AD. Although it has previously been reported that a significant negative effect of education was only found in the right cornus ammonis (CA)1 region of the hippocampus, the authors did not consider the variable of diagnosis when examining the effect of education on the hippocampal subfield volumes by linear regression. ${ }^{17}$ Furthermore, although aMCI subtype has been reported to represent a prodromal stage of Alzheimer's disease, ${ }^{18}$ this previous study did not distinguish between amnestic aMCI patients and non-amnestic aMCI patients.

The objective of this study was to explore the association of education with hippocampal subfield volumes while controlling for multiple variables including diagnosis, age, sex, and APOE $\varepsilon 4$ genotype in healthy control and aMCI groups. In this study, we evaluated the degree of education as education years, which can be quantified objectively. Furthermore, our study used an automated segmentation method built with a novel atlasing algorithm and ex vivo MRI data from autopsy brains, which has been shown to be more accurate than the automated segmentation methods used in previous studies. ${ }^{19}$ Moreover, we attempted to explore the relationship between hippocampal subfield volumes, education, and amnestic cognitive functions in healthy controls and aMCI patients.

\section{Subjects and methods Subjects}

Seventy-seven subjects were included in this study (38 with aMCI [age range: 63-89 years] and 39 healthy elderly controls [age range: $62-87$ years]). The cognitive functions of all the subjects were assessed with the Korean version of Consortium to Establish a Registry for Alzheimer's Disease (CERAD-K), including Verbal Fluency, 15-item Boston Naming Test, the Korean version of mini mental status examination (MMSE-K), ${ }^{20}$ word list memory (WLM), word list recall (WLR), word list recognition (WLRc), Constructional Praxis, and constructional recall (CR). ${ }^{21}$ Patients with aMCI met Petersen's criteria: ${ }^{18} 1$ ) memory complaint corroborated by an informant; 2) objective memory impairment for age, education, and sex; 3 ) essentially preserved general cognitive function; 4) largely intact functional activities; and 5) no dementia. All aMCI patients had an overall Clinical Dementia Rating of $0.5 .{ }^{22}$ Objective memory impairment was defined as a performance score of 1.5 SDs below the respective age-specific, education-specific, and sex-specific normative means on at least one of the four episodic memory tests included in the CERAD-K, namely, the WLM, WLR, WLRc, and CR tests. ${ }^{21}$ Performance scores of other nonamnestic cognitive tests were 1.5 SDs above the respective age-specific, education-specific, and sex-specific normative means. Concise description of the tests and reviewing process is given in the Supplementary material. We excluded the participants who had any history of alcoholism, drug abuse, head trauma, psychiatric disorders, or taking any psychotropic medications (eg, cholinesterase inhibitors, antidepressants, benzodiazepines, and antipsychotics).

The inclusion criteria of healthy elderly controls were as follows: 1 ) subjects aged $>60$ years; 2 ) performance scores of eight tests in the CERAD-K battery were 1.5 SDs above the respective age-specific, education-specific, and sex-specific normative means; and 3) Clinical Dementia Rating $=0$. Subjects with any history of alcoholism, drug abuse, head trauma, psychiatric disorders, or taking any psychotropic medications were excluded. The study was conducted in accordance with the ethical and safety guidelines set forth by the Institutional Review Board of the Catholic University of Korea. The Institutional Review Board of the Catholic University of Korea approved all study procedures. Written informed consent was obtained from all participants. All participants were right-handed.

\section{MRI acquisition}

All participants underwent MRI scans on a $3 \mathrm{~T}$ whole-body scanner equipped with an eight-channel phased-array head coil (Verio; Siemens, Erlangen, Germany). The scanning parameters of the T1-weighted three-dimensional magnetization-prepared rapid gradient-echo sequences 
were as follows: $\mathrm{TE}=2.2 \mathrm{~ms}$, $\mathrm{TR}=1,780 \mathrm{~ms}$, inversion time $=900 \mathrm{~ms}$, flip angle $=9^{\circ}$, field of view $=250 \times 250 \mathrm{~mm}^{2}$, matrix $=256 \times 256$, and voxel size $=1.0 \times 1.0 \times 1.0 \mathrm{~mm}^{3}$.

\section{Image processing}

For surface and volumetric segmentation of the whole brain, we used the FreeSurfer image analysis suite (version 6.0, http://surfer.nmr.mgh.harvard.edu). ${ }^{19}$ The technical details of these procedures have been described in previous publications. ${ }^{23,24}$ Briefly, the processing stream included a Talairach transformation of each subject's native brain, removal of non-brain tissue, and segmentation of the gray matter/white matter tissue. The cortical surface of each hemisphere was inflated to an average spherical surface to locate both the pial surface and the gray matter/white matter boundary.

Hippocampal volumes were obtained from the automated procedure for volumetric measures of brain structures implemented in FreeSurfer. Automated segmentation of the hippocampus to its respective subfields was performed using Bayesian inference, a statistical model of the medial temporal lobe and an ex vivo atlas. The Dice overlap measures between the manual and automated segmentation methods were $\sim 0.7$ for all substructures. ${ }^{16} \mathrm{We}$ focused on the volume of the CA1, CA3, CA4, granule cell layer of dentate gyrus (GC-DG), parasubiculum, presubiculum, and subiculum and excluded the fimbria (which is a white matter region), hippocampal fissure, and the final portion of the hippocampal tail (which is not subdividable in any of the subfields) from the analyses. Total hippocampal volume was computed by summing the relative volumes of the CA3, CA4, GC-DG, parasubiculum, presubiculum, subiculum, and hippocampal tail (Figure 1).

\section{Statistical analyses}

Statistical analyses for demographic data were performed with R software (version 2.15.3). ${ }^{25}$ Assumptions for normality were tested for all continuous variables using the Kolmogorov-Smirnov test. All variables were normally distributed. The independent $t$-test and the $\chi^{2}$ test were used to assess potential differences between the aMCI and healthy control groups for all demographic variables. All statistical analyses used a two-tailed level of 0.05 for defining statistical significance. In accordance with other volumetric analyses, adjustment was performed for each region by an analysis of covariance approach: adjusted volume = raw volume $-\beta \times($ intracranial volume $[\mathrm{ICV}]-$ mean ICV), where $\beta$ is the slope of the regression of the region of interest volume on ICV. ${ }^{26}$ Adjusted volume was used for all analyses described in this study.

To assess the main effect of education on hippocampal subfield volumes, we used multiple regression analyses adjusting for diagnosis, age, sex, and $A P O E$ genotype. In addition, to evaluate the association of education with the hippocampal subfield volumes in each group, multiple regression analyses were performed in both the healthy

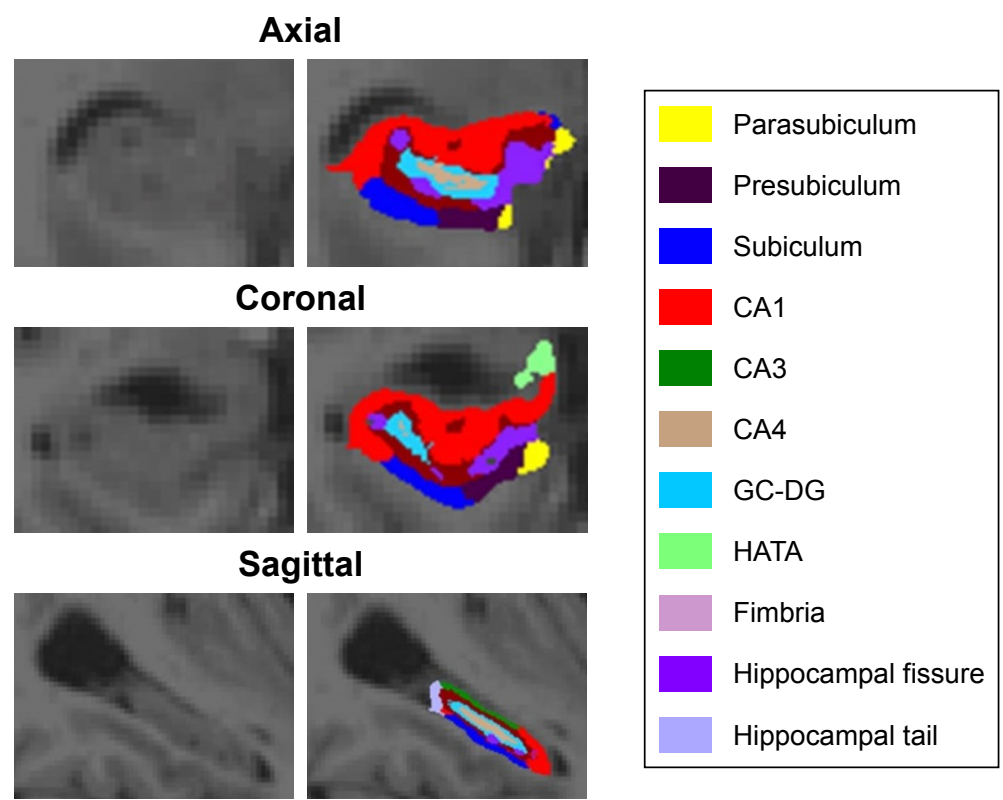

Figure I Illustration of parcellation scheme used for automated hippocampal subfield segmentation: axial view, coronal view, sagittal view. Images are from a control subject. Abbreviations: CA, cornus ammonis; GC-DG, granule cell layer of dentate gyrus; HATA, the hippocampus-amygdala transition area. 
control and aMCI groups, adjusting for age, sex, and $A P O E$ genotype. To assess the relationship between hippocampal subfield volumes and the neuropsychological test scores (MMSE-K, CERAD-K WLM, WLR, and WLRc), multiple regression analyses were performed adjusting for age, sex, education, and $A P O E$ genotype in both the healthy control and aMCI groups. Furthermore, to determine the association between education and the neuropsychological test scores, multiple regression analyses were performed adjusting for age, sex, and $A P O E$ genotype in both the healthy control and aMCI groups. Also, to address the question of whether the hippocampal subfield volumes mediated the association between education and the neuropsychological test scores in the healthy control or aMCI groups, a mediation analysis was performed using the PROCESS macro ${ }^{27}$ controlled for $^{2}$ age, sex, education, and $A P O E$ genotype. All results were considered significant at $p<0.05$.

\section{Results}

\section{Demographic data}

Table 1 shows the baseline demographic data for the different subject groups. There were no significant differences in sex, education, and $A P O E$ genotype between the aMCI and control groups. The control group subjects were significantly younger than the aMCI group subjects $(p<0.05)$. Compared with the control group, the aMCI group showed significantly worse performance on the MMSE-K, CERAD-K WLM, WLR, WLRc, and CR $(p<0.001)$. In regard to non-amnestic cognitive functions, while the aMCI group showed significantly worse performance on the CERAD-K Verbal Fluency than the control group, there was no significant difference in CERAD-K Boston Naming Test and Constructional Praxis scores between the two groups (Table S1). There was no significant difference in total ICV between the aMCI and control groups (Table 1). The detailed clinical characteristics of aMCI group are shown in Table S2.

\section{Relationship between education and hippocampal subfield volumes}

A significant negative correlation with education was found for the left parasubiculum $\left(\beta=-0.26\right.$, adjusted $R^{2}=0.134$, $p<0.01$; Figure S1A), left presubiculum $(\beta=-0.26$, adjusted $R^{2}=0.178, p<0.05$; Figure $\left.\mathrm{S} 1 \mathrm{~B}\right)$, and left subiculum ( $\beta=-0.23$, adjusted $R^{2}=0.256, p<0.05$; Figure $\mathrm{S} 1 \mathrm{C}$ ) in both groups (Table 2 ). The negative correlation of education with left parasubiculum $(\beta=-0.37, p<0.05$; Figure S1D), left presubiculum $(\beta=-0.28, p=0.055)$, and left subiculum volume $(\beta=-0.31, p<0.05$; Figure S1E) showed significance and a trend toward significance in the aMCI group (Table 2). None of the hippocampal subfields were significantly correlated with education in the control group ( $p>0.05$; Table 2$)$.

\section{Relationship between hippocampal subfield volumes and neuropsychological test scores}

A significant negative correlation was found between left/ right parasubiculum, left/right presubiculum, left subiculum volume, and CERAD-K WLR scores in the control group (Table $3 \mathrm{C}$ ). In particular, the strongest relationship was found in the left parasubiculum $(\beta=-0.65, p=0.001$; Figure S2A) and presubiculum ( $\beta=-0.64, p=0.001$; Figure S2B $)$. A significant positive relationship was also found between left CA1, left CA3, left/right CA4, left subiculum, left/right total hippocampus, and CERAD-K WLRc scores in the control group (Table 3D). Particularly, the strongest correlation was found in the left CA3 ( $\beta=-0.65, p=0.001$; Figure $\mathrm{S} 2 \mathrm{C})$ and

Table I Demographic and clinical characteristics of the study participants

\begin{tabular}{|c|c|c|c|}
\hline & Control group $(n=39)$ & aMCl group $(n=38)$ & $p$-value \\
\hline Age (years) & $71.8 \pm 5.8(62-87)$ & $75.5 \pm 5.4(63-89)$ & 0.006 \\
\hline Sex (M:F, \%) & $46: 54$ & $26: 74$ & 0.116 \\
\hline Education (years) & $9.1 \pm 6.3(0-21)$ & $10.2 \pm 4.7(0-20)$ & 0.368 \\
\hline APOE4 carrier (n, \%) & $6(18.2 \%)$ & $9(25.7 \%)$ & 0.648 \\
\hline MMSE-K & $26.5 \pm 2.4(23-30)$ & $23.1 \pm 3.9(I I-29)$ & $<0.001$ \\
\hline CERAD-K WLM & $17.3 \pm 3.9(10-28)$ & $12.9 \pm 3.9(6-20)$ & $<0.001$ \\
\hline CERAD-K WLR & $5.5 \pm 1.6(3-9)$ & $2.7 \pm 1.8(0-8)$ & $<0.001$ \\
\hline CERAD-K WLRc & $8.9 \pm 1.2(6-1 I)$ & $6.5 \pm 2.4(1-10)$ & $<0.001$ \\
\hline CERAD-K CR & $7.1 \pm 2.9(2-1 \mathrm{I})$ & $3.7 \pm 2.5(0-8)$ & $<0.001$ \\
\hline $\operatorname{TICV}\left(\mathrm{mm}^{3}\right)$ & $\begin{array}{l}I, 445,879.9 \pm 162,780.2 \\
(I, 099,427-1,727,122)\end{array}$ & $\begin{array}{l}1,453,963.5 \pm 166,234.4 \\
(I, I 5 I, 643-1,786,046)\end{array}$ & 0.830 \\
\hline
\end{tabular}

Note: Data are means \pm SD (minimum-maximum) unless indicated otherwise.

Abbreviations: $\mathrm{AMCl}$, amnestic mild cognitive impairment; CERAD-K, the Korean version of Consortium to Establish a Registry for Alzheimer's Disease; CR, constructional recall; MMSE-K, the Korean version of mini mental status examination; TICV, total intracranial volume; WLM, word list memory; WLR, word list recall; WLRc, word list recognition. 
Table 2 Relationship between hippocampal subfield volumes and education

\begin{tabular}{|c|c|c|c|c|c|c|c|c|c|}
\hline \multirow[t]{2}{*}{ Hippocampus } & \multicolumn{3}{|c|}{ Education years ${ }^{a}$ (both groups) } & \multicolumn{3}{|c|}{ Education years ${ }^{\mathrm{b}}$ (control group) } & \multicolumn{3}{|c|}{ Education years ${ }^{\mathrm{b}}$ (aMCl group) } \\
\hline & Std. $\beta$ & $t$ & $p$-value & Std. $\beta$ & $t$ & $p$-value & Std. $\beta$ & $t$ & $p$-value \\
\hline \multicolumn{10}{|l|}{ Left } \\
\hline CAI & -0.06 & -0.53 & 0.6 & 0.22 & 1.12 & 0.27 & -0.22 & -1.29 & 0.206 \\
\hline CA3 & -0.13 & -1.04 & 0.305 & 0.05 & 0.23 & 0.823 & -0.16 & -0.91 & 0.372 \\
\hline CA4 & -0.1 & -0.84 & 0.404 & 0.15 & 0.70 & 0.489 & -0.21 & -1.28 & 0.211 \\
\hline GC-DG & -0.11 & -0.95 & 0.348 & 0.14 & 0.67 & 0.507 & -0.23 & -1.43 & 0.163 \\
\hline Parasubiculum & -0.31 & -2.70 & 0.009 c,\# & -0.29 & -1.36 & 0.185 & -0.37 & -2.43 & $\left.0.02\right|^{\mathrm{c}, *}$ \\
\hline Presubiculum & -0.26 & -2.38 & $0.02^{\mathrm{c}, *}$ & -0.15 & $-0.7 \mid$ & 0.485 & -0.28 & -2.00 & $0.055^{c}$ \\
\hline Subiculum & -0.23 & -2.19 & $0.032^{c, *}$ & -0.11 & -0.56 & 0.579 & -0.31 & -2.12 & $0.042^{c, *}$ \\
\hline Total & -0.19 & -1.72 & $0.09^{c}$ & 0.05 & 0.26 & 0.797 & -0.26 & -1.68 & $0.102^{c}$ \\
\hline \multicolumn{10}{|l|}{ Right } \\
\hline CAI & -0.11 & -1.13 & 0.371 & 0.18 & 0.92 & 0.365 & -0.13 & -0.82 & 0.42 \\
\hline $\mathrm{CA} 3$ & -0.11 & -0.84 & 0.403 & 0.12 & 0.61 & 0.547 & -0.08 & -0.98 & 0.336 \\
\hline CA4 & -0.05 & -0.39 & 0.698 & 0.29 & 1.43 & 0.164 & -0.18 & -1.04 & 0.305 \\
\hline GC-DG & -0.05 & -0.64 & 0.705 & 0.34 & 1.83 & $0.077^{c}$ & -0.17 & -1.06 & 0.296 \\
\hline Parasubiculum & -0.2 & -1.69 & $0.095^{c}$ & -0.13 & -0.61 & 0.549 & -0.29 & -1.83 & $0.077^{c}$ \\
\hline Presubiculum & -0.15 & -1.22 & 0.229 & 0.01 & 0.02 & 0.98 & -0.21 & -1.29 & 0.208 \\
\hline Subiculum & -0.17 & -1.53 & $0.132^{c}$ & 0.02 & 0.12 & 0.907 & -0.22 & -1.48 & $0.149^{c}$ \\
\hline Total & -0.1 & -0.83 & 0.41 & 0.19 & 0.99 & 0.329 & -0.19 & -1.16 & 0.257 \\
\hline
\end{tabular}

Notes: aSubjects' age, sex, diagnosis and APOE $\varepsilon 4$ genotype were used as covariates. 'Subjects' age, sex, and APOE $\varepsilon 4$ genotype were used as covariates, 'Education was finally selected as an independent variable after performing stepwise regression $\left({ }^{*} p<0.05 ;{ }^{*} p<0.01\right)$.

Abbreviations: aMCl, amnestic mild cognitive impairment; CA, cornus ammonis; GC-DG, granule cell layer of dentate gyrus; std. $\beta$, standardized beta.

the left CA4 ( $\beta=-0.65, p=0.001$; Figure S2D) in the control group. In addition, a significant positive relationship was found between left/right GC-DG, right CA4, right presubiculum, right total hippocampus, and CERAD-K CR scores in the aMCI group. The strongest association was found in the right presubiculum ( $\beta=0.45, p=0.005$; Figure $\mathrm{S} 2 \mathrm{E}$ ). No hippocampal subfield volumes were significantly correlated with the MMSE-K and CERAD-K WLM scores in both the control and aMCI groups ( $p>0.05$; Table $3 \mathrm{~A}, \mathrm{~B}$, and $\mathrm{E})$.

\section{Relationship between education and neuropsychological test scores}

A significant positive correlation was found between education and CERAD-K CR in both the control $(\beta=0.74, p<0.001$; Table 4) and aMCI groups $(\beta=0.45, p=0.041$; Table 4$)$.

\section{The mediating effect of hippocampal subfield volumes on the association between education and the neuropsychological test scores}

Given the significant relationship between education, hippocampal subfield volumes, and the CERAD-K CR scores in the aMCI group, a mediation analysis was performed with education as an independent factor and CERAD-K CR scores as dependent factors in the aMCI group. The proposed mediators were the volumes of the left/right GC-DG, right
CA4, and right presubiculum, which showed significant correlation with the CERAD-K CR scores only in the aMCI group. The mediation analysis showed that the effect of education on CERAD-K CR scores was not mediated by the

Table 3 (A-E) Relationships between cognitive functions and hippocampal subfield volumes

A

MMSE-K

\begin{tabular}{|c|c|c|c|c|c|c|}
\hline \multirow[t]{2}{*}{ Hippocampus } & \multicolumn{3}{|c|}{$\begin{array}{l}\text { MMSE-K } \\
\text { (control group) }\end{array}$} & \multicolumn{3}{|c|}{$\begin{array}{l}\text { MMSE-Ka } \\
\text { (aMCl group) }\end{array}$} \\
\hline & Std. $\beta$ & $t$ & $p$-value & Std. $\beta$ & $t$ & $p$-value \\
\hline \multicolumn{7}{|l|}{ Left } \\
\hline CAI & 0.01 & 0.05 & 0.963 & -0.07 & -0.37 & 0.717 \\
\hline CA3 & -0.13 & -0.55 & 0.59 & 0.01 & 0.07 & 0.943 \\
\hline CA4 & -0.13 & -0.55 & 0.585 & 0.02 & 0.11 & 0.911 \\
\hline GC-DG & -0.14 & -0.61 & 0.546 & 0 & 0.01 & 0.989 \\
\hline Parasubiculum & 0.07 & 0.27 & 0.788 & 0.06 & 0.35 & 0.732 \\
\hline Presubiculum & -0.36 & -1.81 & $0.08^{b}$ & 0.04 & 0.24 & 0.816 \\
\hline Subiculum & -0.38 & -2.04 & $0.05^{b}$ & 0.01 & 0.09 & 0.928 \\
\hline Total & -0.19 & -0.83 & 0.415 & -0.03 & -0.16 & 0.876 \\
\hline \multicolumn{7}{|l|}{ Right } \\
\hline CAI & -0.09 & -0.39 & 0.701 & -0.13 & -0.73 & 0.472 \\
\hline $\mathrm{CA} 3$ & 0.24 & 1.03 & 0.313 & -0.02 & -0.09 & 0.927 \\
\hline CA4 & 0.1 & 0.45 & 0.655 & -0.04 & -0.19 & $0.85 I$ \\
\hline GC-DG & 0.07 & 0.33 & 0.744 & -0.03 & -0.19 & 0.849 \\
\hline Parasubiculum & -0.1 & -0.39 & 0.701 & -0.07 & -0.38 & 0.708 \\
\hline Presubiculum & -0.28 & -1.15 & 0.259 & -0.04 & -0.21 & 0.835 \\
\hline Subiculum & -0.3 & -1.30 & 0.206 & -0.03 & -0.16 & 0.878 \\
\hline Total & -0.15 & -0.67 & 0.51 & -0.11 & -0.58 & 0.567 \\
\hline
\end{tabular}

(Continued) 
Table 3 (Continued)

B

CERAD-K word list memory

\begin{tabular}{|c|c|c|c|c|c|c|}
\hline \multirow[t]{2}{*}{ Hippocampus } & \multicolumn{3}{|c|}{$\begin{array}{l}\text { CERAD-K WLMa } \\
\text { (control group) }\end{array}$} & \multicolumn{3}{|c|}{$\begin{array}{l}\text { CERAD-K WLMa } \\
\text { (aMCI group) }\end{array}$} \\
\hline & Std. $\beta$ & $t$ & $p$-value & Std. $\beta$ & $t$ & $p$-value \\
\hline \multicolumn{7}{|l|}{ Left } \\
\hline CAI & 0.26 & $\mathrm{I} .48$ & $0.149^{c}$ & -0.07 & -0.38 & 0.705 \\
\hline CA3 & 0.04 & 0.19 & 0.848 & -0.1 & -0.51 & 0.617 \\
\hline CA4 & 0.33 & 1.95 & $0.06^{c}$ & -0.08 & -0.44 & 0.66 \\
\hline GC-DG & 0.29 & 1.68 & $0.104^{c}$ & -0.05 & -0.26 & 0.796 \\
\hline Parasubiculum & -0.11 & -0.53 & 0.601 & -0.1 & -0.60 & 0.555 \\
\hline Presubiculum & 0.01 & 0.04 & 0.97 & -0.02 & -0.10 & 0.917 \\
\hline Subiculum & 0.26 & 1.58 & $0.124^{c}$ & 0 & 0 & 0.997 \\
\hline Total & 0.14 & 0.74 & 0.464 & -0.05 & -0.32 & 0.752 \\
\hline \multicolumn{7}{|l|}{ Right } \\
\hline CAI & 0.08 & 0.45 & 0.655 & 0.01 & 0.05 & 0.959 \\
\hline CA3 & -0.09 & -0.47 & 0.643 & 0.24 & 1.47 & $0.15 I^{c}$ \\
\hline CA4 & -0.01 & -0.06 & 0.953 & 0.13 & 0.73 & 0.474 \\
\hline GC-DG & -0.01 & -0.08 & 0.936 & 0.14 & 0.82 & 0.421 \\
\hline Parasubiculum & 0.04 & 0.18 & 0.858 & 0.05 & 0.29 & 0.778 \\
\hline Presubiculum & -0.05 & -0.25 & 0.805 & 0.2 & 1.18 & 0.247 \\
\hline Subiculum & -0.01 & -0.04 & 0.97 & 0.08 & 0.50 & 0.62 \\
\hline Total & 0 & 0.02 & 0.986 & 0.07 & 0.39 & 0.696 \\
\hline
\end{tabular}

C

CERAD-K word list recall

\begin{tabular}{|c|c|c|c|c|c|c|}
\hline \multirow[t]{2}{*}{ Hippocampus } & \multicolumn{3}{|c|}{$\begin{array}{l}\text { CERAD-K WLR } \\
\text { (control group) }\end{array}$} & \multicolumn{3}{|c|}{$\begin{array}{l}\text { CERAD-K WLR } \\
\text { (aMCI group) }\end{array}$} \\
\hline & Std. $\beta$ & $t$ & p-value & Std. $\beta$ & $t$ & $p$-value \\
\hline \multicolumn{7}{|l|}{ Left } \\
\hline CAI & 0.15 & 0.66 & 0.516 & 0.02 & 0.12 & 0.909 \\
\hline CA3 & -0.12 & -0.50 & 0.621 & 0.13 & 0.66 & 0.514 \\
\hline CA4 & -0.08 & -0.35 & 0.726 & 0 & 0.00 & I \\
\hline GC-DG & -0.06 & -0.28 & 0.779 & 0.05 & 0.29 & 0.775 \\
\hline Parasubiculum & -0.65 & -3.53 & $0.00 I^{d, \neq}$ & 0.05 & 0.29 & 0.771 \\
\hline Presubiculum & -0.64 & -3.58 & $0.00 I^{d, \neq}$ & -0.09 & -0.57 & 0.576 \\
\hline Subiculum & -0.42 & -2.15 & $0.039^{\mathrm{d}, *}$ & -0.11 & -0.70 & 0.488 \\
\hline Total & -0.27 & -1.37 & $0.183^{d}$ & -0.05 & -0.28 & 0.778 \\
\hline \multicolumn{7}{|l|}{ Right } \\
\hline CAI & -0.09 & -0.41 & 0.688 & -0.16 & -0.93 & 0.362 \\
\hline CA3 & -0.15 & -0.69 & 0.497 & -0.11 & -0.58 & 0.566 \\
\hline CA4 & -0.16 & -0.72 & 0.477 & -0.15 & -0.79 & 0.435 \\
\hline GC-DG & -0.13 & -0.62 & 0.54 & -0.11 & -0.60 & 0.556 \\
\hline Parasubiculum & -0.32 & -1.86 & $0.072^{\mathrm{d}}$ & -0.18 & -1.02 & 0.317 \\
\hline Presubiculum & -0.43 & -2.17 & $0.038^{\mathrm{e}, *}$ & -0.17 & -0.98 & 0.333 \\
\hline Subiculum & $-0.4 \mathrm{I}$ & -2.16 & $0.039 \mathrm{~d}, *$ & -0.14 & -0.80 & 0.431 \\
\hline Total & -0.34 & -1.72 & $0.096^{d}$ & -0.17 & -0.95 & 0.35 \\
\hline
\end{tabular}

D

CERAD-K word list recognition

\begin{tabular}{|c|c|c|c|c|c|c|}
\hline \multirow[t]{2}{*}{ Hippocampus } & \multicolumn{3}{|c|}{$\begin{array}{l}\text { CERAD-K WLRc } \\
\text { (control group) }\end{array}$} & \multicolumn{3}{|c|}{$\begin{array}{l}\text { CERAD-K WLRca } \\
\text { (aMCI group) }\end{array}$} \\
\hline & Std. $\beta$ & $t$ & $p$-value & Std. $\beta$ & $t$ & $p$-value \\
\hline \multicolumn{7}{|l|}{ Left } \\
\hline CAI & 0.48 & 3.20 & $0.003^{f, f}$ & 0.1 & 0.55 & 0.584 \\
\hline $\mathrm{CA} 3$ & 0.57 & 3.84 & $0.00 I^{f, f}$ & 0.06 & 0.33 & 0.747 \\
\hline
\end{tabular}

Table 3 (Continued)

\begin{tabular}{|c|c|c|c|c|c|c|}
\hline \multirow[t]{2}{*}{ Hippocampus } & \multicolumn{3}{|c|}{$\begin{array}{l}\text { CERAD-K WLRc } \\
\text { (control group) }\end{array}$} & \multicolumn{3}{|c|}{$\begin{array}{l}\text { CERAD-K WLRc } \\
\text { (aMCI group) }\end{array}$} \\
\hline & Std. $\beta$ & $t$ & $p$-value & Std. $\beta$ & $t$ & $p$-value \\
\hline CA4 & 0.54 & 3.60 & $0.00 I^{f, q}$ & 0.07 & 0.43 & 0.671 \\
\hline GC-DG & 0.5 & 3.23 & $0.003^{f, f}$ & 0.06 & 0.35 & 0.726 \\
\hline Parasubiculum & -0.01 & -0.07 & 0.948 & 0.06 & 0.40 & 0.69 \\
\hline Presubiculum & 0.12 & 0.2 & 0.548 & -0.02 & -0.16 & 0.878 \\
\hline Subiculum & 0.34 & 2.06 & $0.048^{f * *}$ & -0.03 & -0.20 & 0.843 \\
\hline Total & 0.48 & 3.03 & 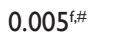 & 0.01 & 0.04 & 0.966 \\
\hline \multicolumn{7}{|l|}{ Right } \\
\hline CAI & 0.32 & 1.97 & $0.059^{f}$ & 0.02 & 0.15 & 0.883 \\
\hline CA3 & 0.28 & 1.60 & $0.121^{f}$ & -0.03 & 0.18 & 0.883 \\
\hline CA4 & 0.36 & 2.26 & $0.032^{f, *}$ & -0.01 & -0.05 & 0.961 \\
\hline GC-DG & 0.27 & 1.66 & $0.108^{f}$ & 0 & 0.02 & 0.982 \\
\hline Parasubiculum & 0.25 & 1.46 & $0.154^{f}$ & -0.03 & -0.20 & 0.844 \\
\hline Presubiculum & 0.32 & 1.91 & $0.065^{f}$ & -0.08 & -0.46 & 0.646 \\
\hline Subiculum & 0.29 & 1.70 & $0 . I^{f}$ & -0.08 & -0.50 & 0.622 \\
\hline Total & 0.37 & 2.30 & $0.028^{f * *}$ & -0.06 & -0.37 & 0.712 \\
\hline
\end{tabular}

CERAD-K constructional recall

\begin{tabular}{lll}
\hline Hippocampus & $\begin{array}{l}\text { CERAD-K CR }^{\mathrm{a}} \\
\text { (control group) }\end{array}$ & $\begin{array}{l}\text { CERAD-K CR }^{\mathrm{a}} \\
\text { (aMCl group) }\end{array}$ \\
\cline { 1 - 2 } & &
\end{tabular}

$\begin{array}{lllll}\text { Std. } \beta & t & p \text {-value } & & \\ \text { Std. } \beta & t & p \text {-value }\end{array}$

\begin{tabular}{|c|c|c|c|c|c|c|}
\hline \multicolumn{7}{|l|}{ Left } \\
\hline CAI & 0.14 & 0.54 & 0.596 & 0.01 & 0.06 & 0.952 \\
\hline CA3 & -0.18 & -0.66 & 0.514 & 0.16 & 0.71 & 0.481 \\
\hline CA4 & -0.11 & -0.42 & 0.677 & 0.18 & 0.84 & 0.405 \\
\hline GC-DG & -0.11 & -0.42 & 0.675 & 0.43 & 2.75 & $\left.0.0\right|^{g, *}$ \\
\hline Parasubiculum & -0.24 & -0.87 & 0.391 & -0.06 & -0.28 & 0.779 \\
\hline Presubiculum & -0.16 & -0.56 & 0.579 & 0.05 & 0.28 & 0.78 \\
\hline Subiculum & -0.02 & -0.07 & 0.945 & 0.18 & 0.95 & 0.349 \\
\hline Total & -0.13 & -0.48 & 0.638 & 0.13 & 0.65 & 0.518 \\
\hline \multicolumn{7}{|l|}{ Right } \\
\hline CAI & -0.05 & -0.21 & 0.834 & 0.07 & 0.32 & 0.755 \\
\hline CA3 & -0.27 & -1.02 & 0.315 & 0.23 & 1.05 & 0.303 \\
\hline CA4 & -0.3 & -1.16 & 0.255 & $0.4 \mathrm{I}$ & 2.59 & $0.014^{g, *}$ \\
\hline GC-DG & -0.26 & -1.08 & 0.288 & 0.4 & 2.47 & $0.0199 . *$ \\
\hline Parasubiculum & -0.26 & -0.91 & 0.369 & 0.03 & 0.12 & 0.904 \\
\hline Presubiculum & -0.19 & -0.70 & 0.493 & 0.45 & 2.98 & $0.005^{g, A}$ \\
\hline Subiculum & -0.07 & -0.26 & 0.797 & 0.26 & 1.30 & 0.205 \\
\hline Total & -0.2 & -0.79 & 0.434 & 0.41 & 2.60 & $0.0148, *$ \\
\hline
\end{tabular}

Notes: a'Subjects' age, sex, education, and APOE \&4 genotype were used as covariates. 'MMSE- $\mathrm{K}$ was finally selected as an independent variable after performing stepwise regression. 'CERAD-K word list memory was finally selected as an independent variable after performing stepwise regression. ${ }^{\circ} \mathrm{CERAD}-\mathrm{K}$ word list recall was finally selected as an independent variable after performing stepwise regression. elnsignificant finally selected model after performing stepwise regression. 'CERAD-K word list recognition was finally selected as an independent variable after performing stepwise regression. ${ }^{8} \mathrm{CERAD}-\mathrm{K}$ constructional recall was finally selected as an independent variable after performing stepwise regression. ${ }^{*} p<0.05 ;{ }^{*} p<0.0$ I; ${ }^{\ddagger} p<0.005$.

Abbreviations: $\mathrm{aMCl}$, amnestic mild cognitive impairment; $\mathrm{CA}$, cornus ammonis; GC-DG, granule cell layer of dentate gyrus; MMSE-K, the Korean version of mini mental status examination; std. $\beta$, standardized beta; CERAD-K, the Korean version of Consortium to Establish a Registry for Alzheimer's Disease; WLRc, word list recognition; WLR, word list recall. 
Table 4 Relationships between cognitive functions and education

\begin{tabular}{|c|c|c|c|c|c|c|}
\hline \multirow[t]{2}{*}{ Cognitive function } & \multicolumn{3}{|c|}{ Education years ${ }^{a}$ (control group) } & \multicolumn{3}{|c|}{ Education years ${ }^{\mathrm{a}}$ (aMCl group) } \\
\hline & Std. $\beta$ & $t$ & $p$-value & Std. $\beta$ & $t$ & $p$-value \\
\hline MMSE-K & 0.45 & 3.08 & $0.004^{\mathrm{b}, \neq}$ & 0.46 & 2.85 & $0.008^{\mathrm{b}, \#}$ \\
\hline CERAD-K word list memory & 0.32 & 1.60 & $0.121^{b}$ & 0.16 & 0.96 & 0.346 \\
\hline CERAD-K word list recall & 0.23 & 1.21 & 0.237 & 0.09 & 0.53 & 0.602 \\
\hline CERAD-K word list recognition & 0.02 & 0.08 & 0.936 & 0.04 & 0.25 & 0.807 \\
\hline CERAD-K constructional recall & 0.74 & 6.04 & $<0.00 \mathrm{I}^{\mathrm{b}, \mathrm{f}}$ & 0.3 & 2.14 & $\left.0.04\right|^{\mathrm{b}, *}$ \\
\hline
\end{tabular}

Notes: aSubjects' age, sex, and APOE $\varepsilon 4$ genotype were used as covariates. 'Education years were finally selected as an independent variable after performing stepwise regression $\left({ }^{*} p<0.05 ;{ }^{*} p<0.01 ;{ }^{*} p<0.005\right)$.

Abbreviations: aMCl, amnestic mild cognitive impairment; CERAD-K, the Korean version of Consortium to Establish a Registry for Alzheimer's Disease; MMSE-K, the Korean version of mini mental status examination; std. $\beta$, standardized beta.

volumes of any aforementioned hippocampal subfields in the aMCI group (Results section in Supplementary material).

\section{Discussion}

We initially examined the association of education with hippocampal subfield volumes in cognitively normal older adults and aMCI patients. Although total hippocampal volume showed nonsignificant association with education, the left parasubiculum, left presubiculum, and left subiculum volumes exhibited a significant negative correlation with education. However, these findings do not support previous studies which demonstrated a negative correlation of education with the right $\mathrm{CA} 1$ volume in a combined cohort group of healthy controls and patients with MCI and AD. ${ }^{17}$ This discrepancy could be attributed to the exclusion of diagnosis as an independent variable in the multiple linear regression analysis in the previous study. ${ }^{17} \mathrm{~A}$ diagnosis of aMCI has been demonstrated to be associated with smaller hippocampal subfield volumes including the subiculum, presubiculum, and CA1-CA2 transition zone. ${ }^{13,15}$ In this respect, exclusion of diagnosis as an independent variable in multiple linear regressions could have affected the relationship between education and the hippocampal subfield volumes in the previous study. Furthermore, given that amnestic and nonamnestic cognitive functions have attributed to the different laterality of hippocampus, ${ }^{28,29}$ the fact that the previous study did not distinguish amnestic MCI patients from non-amnestic MCI patients could have affected the inconsistency. Another explanation for this discrepancy might be the heterogeneity of the aMCI group. Individuals in the amnestic MCI group could have been classified as late MCI or early MCI based on CERAD-K delayed recall test scores (Methods section in Supplementary material). ${ }^{30}$ Results from an earlier study demonstrated a significant difference in left hippocampal volume between late MCI and early MCI groups. ${ }^{30}$ In our study, the percentage of subjects with early MCI was higher than that of late $\mathrm{MCI}(55.3 \%$ vs $44.7 \%$; Table S2 $)$. Therefore, the heterogeneous aMCI group in our study could have had a confounding effect on these results. Another possible explanation for the discrepancy is the lack of data on beta-amyloid (A $\beta$ ) retention in this study. It has been reported that aMCI patients with $A \beta$ retention showed smaller hippocampal volumes than those without $A \beta$ retention. ${ }^{31}$ Furthermore, $A \beta$ retention has been demonstrated to have a significant interaction with cognitive reserve proxies (including education) on the volume of the left hippocampus. ${ }^{32}$ Therefore, $A \beta$ retention could affect hippocampal subfield volumes and may have had a confounding effect on the current results.

Another important finding was that only the aMCI group showed a significance in a negative relationship between education and left parasubiculum/subiculum volumes. Cognitive reserve including the level of education has been demonstrated to have a negative association with brain volumes, including hippocampus, in MCI and $\mathrm{AD}$ patients compared to healthy controls. ${ }^{33}$ A possible explanation for this might be that individuals with a high cognitive reserve could have increased AD pathology after clinical presentation due to a higher tolerance to $\mathrm{AD}$ pathology, and this increased $\mathrm{AD}$ pathology could affect the brain structure in $\mathrm{MCI}$ and $\mathrm{AD}$ patients. ${ }^{34}$ In addition, the laterality of hippocampus was found in the relationship between education and hippocampal subfield volumes. Although there have been no controlled studies which explore the effect of education on the laterality of hippocampus, the asymmetry of hippocampus has been reported to be critical for the appropriate memory acquisition and retention, ${ }^{28}$ being associated with integration of the verbal and nonverbal memory. ${ }^{35} \mathrm{In}$ these regards, our findings might be interpreted with the aforementioned propositions.

Both the subiculum, which correlated with education in this study, and the CA1, which correlated with education in a previous study, have been suggested to provide the dominant outflow of the hippocampal circuit consisting of hippocampal subfields. ${ }^{36}$ The outflow of these subregions has been reported 
to connect with the medial prefrontal, orbitofrontal, and anterior and posterior cingulate cortices, ${ }^{37,38}$ which are default mode network and compensatory network regions..$^{8,39,40}$ In cognitively normal older adults and $\mathrm{AD}$ patients, cognitive reserve can recruit new and compensatory brain networks to function against advancing $\mathrm{AD}$ pathogenesis. ${ }^{41}$ Therefore, the findings of this and previous studies raise intriguing questions regarding the role of hippocampal subfields such as the subiculum and CA1 in providing compensatory mechanisms permitted by cognitive reserve, considering that education has been documented to be a standard proxy of cognitive reserve. ${ }^{42}$

The second aim of this study was to explore the relationship between hippocampal subfield volumes, education, and amnestic cognitive functions in both the healthy control and aMCI groups. We found that the left presubiculum and parasubiculum volumes were most significantly associated with CERAD-K WLR scores in the control group, the left CA3 and CA4 volumes were most significantly correlated with the CERAD-K WLRc scores in the control group, and that the right presubiculum volume was most significantly related with the CERAD-K CR scores in the aMCI group. Although there have been few studies on the relationships between the hippocampal subfield volumes and the neuropsychological tests in the healthy control and aMCI groups, an automated hippocampal shape analysis method has displayed a positive association between CERAD WLR scores and deformation of the CA1 and subiculum in aMCI subjects. ${ }^{43}$ Furthermore, our previous study has demonstrated significant correlations between left presubiculum, subiculum volumes and the MMSE-K/CERAD-K WLR scores in AD subjects, but not in healthy older controls. ${ }^{44}$ However, the current findings are limited by a paucity of existing evidence on the relationship between the hippocampal subfield volumes and the amnestic cognitive functions measured by cognitive assessment battery in the progression of AD. Furthermore, the aforementioned limitations could have also affected this relationship in a similar way. Notwithstanding these limitations, this finding offers some insight into the laterality of hippocampus. In this study, whereas verbal amnestic cognitive functions were most significantly associated with the left hippocampal subfield volumes, nonverbal amnestic cognitive functions were most significantly correlated with the right hippocampal subfield volumes. This is in accordance with previous observations that mnemonic functions for verbal and nonverbal stimuli are distinguishable and asymmetrically recruit the left and right hippocampus, respectively. ${ }^{29,35}$ Additionally, these results must be interpreted with caution because the degree of cognitive decline is not substantial in the early phase of $\mathrm{AD}$, restricting the relationship between the hippocampal subfield volumes and the amnestic cognitive functions.

In functional MRI studies, the subiculum is observed to have differential brain activity during the retrieval of hippocampus-dependent memories ${ }^{45-48}$ and has been associated with clock drawing test score in aMCI and AD patients. ${ }^{49}$ In animal studies, the subiculum is downstream of the deep layer of the entorhinal cortex, ${ }^{12}$ which was found to be involved in delayed retention. ${ }^{50}$ Hence, it is possible that the subiculum volume might be associated with memorybased neuropsychological test scores. In addition, the presubiculum, a substructure of the subiculum, has also been reported to be correlated with clock drawing test score in AD patients. ${ }^{48}$ In contrast to the subiculum, few studies are available that supply evidence allowing inferences about the relationship between the presubiculum and amnestic cognitive functions. Given that the presubiculum is a substructure of the subiculum, further work is required to fully examine the role of the presubiculum in amnestic cognitive function. The CA3 has also been demonstrated to participate in recognition memory, along with GC-DG. ${ }^{51}$ Therefore, these previous results broadly support our current observations on the relationship between the hippocampal subfield volumes and the amnestic cognitive functions.

The mediation analysis revealed nonsignificant indirect effect of hippocampal subfields on the relationship between education and amnestic cognitive function. As the functional changes of hippocampus have been documented to be correlated with cognitive functions, ${ }^{50,52}$ additional studies will be needed to develop an integrated understanding of the hippocampal subfields by evaluating both functional and structural changes of the hippocampal subfields.

The principal limitation of this study was the lack of a high-resolution T2 scan for automated hippocampus segmentation. It has been reported that automated segmentation using combined T1/T2 data better captures differences in the subregions between healthy controls and AD patients, compared to using only $\mathrm{T} 1$ scans. ${ }^{19}$ In addition, although studies were conducted on aMCI patients, the combination of single domain and multiple domain aMCI subjects may have affected the outcome, given the differential effect of this categorization on the conversion of $\mathrm{AD} .{ }^{53}$ Therefore, future studies should attempt to minimize bias by using combined T1/T2 data, including a larger sample of subjects, and considering confounding factors such as detailed classification of aMCI and the presence of $\mathrm{A} \beta$ retention.

Although these findings should be interpreted with caution, this study has several strengths including the use of automated segmentation of the hippocampus with improved 
accuracy and robust statistical analysis while controlling for clinically related variables to evaluate the associations between education, hippocampal subfield volumes, and amnestic cognitive functions. An additional strength is that this study focused on the early phases of AD. Hippocampal atrophy has been known to affect the transition from normality to mild cognitive impairment. ${ }^{54}$ Moreover, cognitive reserve has been demonstrated to be correlated with functional brain reorganization in the parietotemporal regions during the early course of AD. ${ }^{33}$ In this respect, focusing on the early phase of AD might allow the effect of education on hippocampal subfield volumes to be examined more robustly.

This study identified a negative association of education with presubiculum and subiculum volumes after adjusting for multiple variables including diagnosis, age, sex, and $A P O E \varepsilon 4$ genotype. The second major finding was that the parasubiculum, presubiculum, CA3 and CA4 volumes were associated with mnemonic neuropsychological test scores in both groups. This study adds to the growing body of research indicating that cognitive reserve could impact hippocampal subfields and related amnestic cognitive functions in the early phase of $\mathrm{AD}$. In addition, further research with other cognitive reserve proxies is required to better understand the complex interaction of cognitive reserve and the pathogenesis of $\mathrm{AD}$.

\section{Acknowledgment}

This research was supported by the Ministry of Trade, Industry and Energy (MOTIE, Korea) under Industrial Technology Innovation Program No 10062378.

\section{Author contributions}

DWK, HKL, and CUkL conceived and designed the research. DWK, HKL, S-hJ, and CUL recruited subjects and processed them to get clinical results. DWK, NRL, and HKL performed the in vivo MRI experiments. DWK, NRL, and HKL performed the image preprocessing and image analysis. NRL and SHJ performed statistical analysis. DWK, HKL, and SHJ wrote the manuscript. CUkL provided scientific mentorship throughout the project. All authors discussed the results and critically revised the manuscript for important intellectual content.

\section{Disclosure}

The authors report no conflicts of interest in this work.

\section{References}

1. Barnes DE, Yaffe K. The projected effect of risk factor reduction on Alzheimer's disease prevalence. Lancet Neurol. 2011;10(9):819-828.

2. Zhang M, Katzman R, Salmon D, et al. The prevalence of dementia and Alzheimer's disease in Shanghai, China: impact of age, gender, and education. Ann Neurol. 1990;27(4):428-437.
3. Meng X, D'Arcy C. Education and dementia in the context of the cognitive reserve hypothesis: a systematic review with meta-analyses and qualitative analyses. PLoS One. 2012;7(6):e38268.

4. Squire LR, Zola-Morgan S. The medial temporal lobe memory system. Science. 1991;253(5026):1380.

5. Braak H, Braak E. Neuropathological stageing of Alzheimer-related changes. Acta Neuropathol. 1991;82(4):239-259.

6. Piras F, Cherubini A, Caltagirone C, Spalletta G. Education mediates microstructural changes in bilateral hippocampus. Hum Brain Mapp. 2011;32(2):282-289.

7. Scarmeas N, Zarahn E, Anderson KE, et al. Association of life activities with cerebral blood flow in Alzheimer disease: implications for the cognitive reserve hypothesis. Arch Neurol. 2003;60(3):359-365.

8. Wang L, Zang Y, He Y, et al. Changes in hippocampal connectivity in the early stages of Alzheimer's disease: evidence from resting state fMRI. Neuroimage. 2006;31(2):496-504.

9. Eckerström C, Olsson E, Borga M, et al. Small baseline volume of left hippocampus is associated with subsequent conversion of MCI into dementia: the Göteborg MCI study. J Neurol Sci. 2008; 272(1):48-59.

10. Lo RY, Jagust WJ. Effect of cognitive reserve markers on Alzheimer pathological progression. Alzheimer Dis Assoc Dis. 2013;27(4):343-350.

11. Valenzuela MJ, Sachdev P, Wen W, Chen X, Brodaty H. Lifespan mental activity predicts diminished rate of hippocampal atrophy. PLoS One. 2008;3(7):e2598.

12. Small SA, Schobel SA, Buxton RB, Witter MP, Barnes CA. A pathophysiological framework of hippocampal dysfunction in ageing and disease. Nat Rev Neurosci. 2011;12(10):585-601.

13. Mueller SG, Schuff N, Yaffe K, Madison C, Miller B, Weiner MW. Hippocampal atrophy patterns in mild cognitive impairment and Alzheimer's disease. Hum Brain Mapp. 2010;31(9):1339-1347.

14. Mueller SG, Weiner MW. Selective effect of age, Apo e4, and Alzheimer's disease on hippocampal subfields. Hippocampus. 2009;19(6):558-564.

15. Carlesimo GA, Piras F, Orfei MD, Iorio M, Caltagirone C, Spalletta G. Atrophy of presubiculum and subiculum is the earliest hippocampal anatomical marker of Alzheimer's disease. Alzheimers Dement. 2015; $1(1): 24-32$.

16. Van Leemput K, Bakkour A, Benner T, et al. Automated segmentation of hippocampal subfields from ultra-high resolution in vivo MRI. Hippocampus. 2009;19(6):549-557.

17. Khan W, Westman E, Jones N, et al. Automated hippocampal subfield measures as predictors of conversion from mild cognitive impairment to Alzheimer's disease in two independent cohorts. Brain Topogr. 2015; 28(5):746-759.

18. Petersen RC. Mild cognitive impairment as a diagnostic entity. J Intern Med. 2004;256(3):183-194.

19. Iglesias JE, Augustinack JC, Nguyen K, et al. A computational atlas of the hippocampal formation using ex vivo, ultra-high resolution MRI: application to adaptive segmentation of in vivo MRI. Neuroimage. 2015; 115:117-137.

20. Park J. Standardization of Korean of the mini-mental state examination (mmse-k) for use in the elderly. Part II. Diagnostic validity. J Korean Neuropsychiatr Assoc. 1989;28:508-513.

21. Lee JH, Lee KU, Lee DY, et al. Development of the korean version of the consortium to establish a registry for Alzheimer's disease assessment packet (CERAD-K) clinical and neuropsychological assessment batteries. J Gerontol Ser B. 2002;57(1):P47-P53.

22. Morris JC. The clinical dementia rating (CDR): current version and scoring rules. Neurology. 1993;43(11):2412-2414.

23. Dale AM, Fischl B, Sereno MI. Cortical surface-based analysis: I. Segmentation and surface reconstruction. Neuroimage. 1999;9(2): 179-194.

24. Fischl B, Dale AM. Measuring the thickness of the human cerebral cortex from magnetic resonance images. Proc Natl Acad Sci. 2000; 97(20):11050-11055.

25. Team R. R Development Core Team: R: A Language and Environment for Statistical Computing. Vienna: R Foundation for Statistical Computing; 2011. 
26. Raz N, Lindenberger U, Rodrigue KM, et al. Regional brain changes in aging healthy adults: general trends, individual differences and modifiers. Cereb Cortex. 2005;15(11):1676-1689.

27. Preacher KJ, Hayes AF. Asymptotic and resampling strategies for assessing and comparing indirect effects in multiple mediator models. Behav Res Methods. 2008;40(3):879-891.

28. Goto K, Kurashima R, Gokan H, Inoue N, Ito I, Watanabe S. Left-right asymmetry defect in the hippocampal circuitry impairs spatial learning and working memory in iv mice. PLoS One. 2010;5(11):e15468.

29. Dalton MA, Hornberger M, Piguet O. Material specific lateralization of medial temporal lobe function: an fMRI investigation. Hum Brain Mapp. 2016;37(3):933-941.

30. Zhuang L, Sachdev PS, Trollor JN, et al. Microstructural white matter changes, not hippocampal atrophy, detect early amnestic mild cognitive impairment. PLoS One. 2013;8(3):e58887.

31. Huijbers W, Mormino EC, Schultz AP, et al. Amyloid- $\beta$ deposition in mild cognitive impairment is associated with increased hippocampal activity, atrophy and clinical progression. Brain. 2015;138(4): 1023-1035.

32. Arenaza-Urquijo EM, Molinuevo J-L, Sala-Llonch R, et al. Cognitive reserve proxies relate to gray matter loss in cognitively healthy elderly with abnormal cerebrospinal fluid amyloid- $\beta$ levels. $J$ Alzheimers Dis. 2013;35(4):715-726.

33. Solé-Padullés C, Bartrés-Faz D, Junqué C, et al. Brain structure and function related to cognitive reserve variables in normal aging, mild cognitive impairment and Alzheimer's disease. Neurobiol Aging. 2009; 30(7):1114-1124.

34. Bartrés-Faz D, Arenaza-Urquijo EM. Structural and functional imaging correlates of cognitive and brain reserve hypotheses in healthy and pathological aging. Brain Topogr. 2011;24(3-4):340.

35. Iglói K, Doeller CF, Berthoz A, Rondi-Reig L, Burgess N. Lateralized human hippocampal activity predicts navigation based on sequence or place memory. Proc Natl Acad Sci U S A. 2010;107(32): 14466-14471.

36. Van Strien NM, Cappaert N, Witter MP. The anatomy of memory: an interactive overview of the parahippocampal-hippocampal network. Nat Rev Neurosci. 2009;10(4):272.

37. Rosene DL, Van Hoesen GW. Hippocampal efferents reach widespread areas of cerebral cortex and amygdala in the rhesus monkey. Science. 1977;198(4314):315-317.

38. Kobayashi Y, Amaral DG. Macaque monkey retrosplenial cortex: II. Cortical afferents. J Comp Neurol. 2003;466(1):48-79.

39. Grady CL, McIntosh AR, Beig S, Keightley ML, Burian H, Black SE. Evidence from functional neuroimaging of a compensatory prefrontal network in Alzheimer's disease. J Neurosci. 2003;23(3):986-993.
40. Gould R, Brown R, Owen A, Howard R. fMRI BOLD response to increasing task difficulty during successful paired associates learning. Neuroimage. 2003;20(2):1006-1019.

41. Stern Y. Cognitive reserve. Neuropsychologia. 2009;47(10):2015-2028.

42. Stern Y, Alexander GE, Prohovnik I, Mayeux R. Inverse relationship between education and parietotemporal perfusion deficit in Alzheimer's disease. Ann Neurol. 1992;32(3):371-375.

43. Costafreda SG, Dinov ID, Tu Z, et al. Automated hippocampal shape analysis predicts the onset of dementia in mild cognitive impairment. Neuroimage. 2011;56(1):212-219.

44. Lim H, Hong S, Jung W, et al. Automated segmentation of hippocampal subfields in drug-naive patients with Alzheimer disease. Am J Neuroradiol. 2013;34(4):747-751.

45. Olsen RK, Nichols EA, Chen J, et al. Performance-related sustained and anticipatory activity in human medial temporal lobe during delayed match-to-sample. J Neurosci. 2009;29(38):11880-11890.

46. Zeineh MM, Engel SA, Thompson PM, Bookheimer SY. Dynamics of the hippocampus during encoding and retrieval of face-name pairs. Science. 2003;299(5606):577-580.

47. Gabrieli JD, Brewer JB, Desmond JE, Glover GH. Separate neural bases of two fundamental memory processes in the human medial temporal lobe. Science. 1997;276(5310):264-266.

48. Eldridge LL, Engel SA, Zeineh MM, Bookheimer SY, Knowlton BJ. A dissociation of encoding and retrieval processes in the human hippocampus. J Neurosci. 2005;25(13):3280-3286.

49. Hirjak D, Sambataro F, Remmele B, et al. The relevance of hippocampal subfield integrity and clock drawing test performance for the diagnosis of Alzheimer's disease and mild cognitive impairment. World J Biol Psychiatry. 2017:1-12.

50. Brickman AM, Stern Y, Small SA. Hippocampal subregions differentially associate with standardized memory tests. Hippocampus. 2011; 21(9):923-928.

51. Toner CK, Pirogovsky E, Kirwan CB, Gilbert PE. Visual object pattern separation deficits in nondemented older adults. Learn Mem. 2009; 16(5):338-342.

52. Bakker A, Krauss GL, Albert MS, et al. Reduction of hippocampal hyperactivity improves cognition in amnestic mild cognitive impairment. Neuron. 2012;74(3):467-474.

53. Petersen RC, Doody R, Kurz A, et al. Current concepts in mild cognitive impairment. Arch Neurol. 2001;58(12):1985-1992.

54. Wolf H, Hensel A, Kruggel F, et al. Structural correlates of mild cognitive impairment. Neurobiol Aging. 2004;25(7):913-924.
Neuropsychiatric Disease and Treatment

\section{Publish your work in this journal}

Neuropsychiatric Disease and Treatment is an international, peerreviewed journal of clinical therapeutics and pharmacology focusing on concise rapid reporting of clinical or pre-clinical studies on a range of neuropsychiatric and neurological disorders. This journal is indexed on PubMed Central, the 'PsycINFO' database and CAS,

\section{Dovepress}

and is the official journal of The International Neuropsychiatric Association (INA). The manuscript management system is completely online and includes a very quick and fair peer-review system, which is all easy to use. Visit http://www.dovepress.com/testimonials.php to read real quotes from published authors. 